\title{
Mislocalization-related disease gene discovery using gene expression based computational protein localization prediction
}

\author{
Zhonghao Liu, Jianjun $\mathrm{Hu}^{1, *}$ \\ Department of Computer Science E Engineering, University of South Carolina, 301 Main Street, Columbia, SC, 29208
}

\begin{abstract}
Protein sorting is an important mechanism for transporting proteins to their target subcellular locations after their synthesis. Mutations on genes may disrupt the well regulated protein sorting process, leading to a variety of mislocation related diseases. This paper proposes a methodology to discover such disease genes based on gene expression data and computational protein localization prediction. A kernel logistic regression based algorithm is used to successfully identify several candidate cancer genes which may cause cancers due to their mislocation within the cell. Our results also showed that compared to the gene co-expression network defined on Pearson correlation coefficients, the nonliear Maximum Correlation Coefficients (MIC) based co-expression network give better results for subcellular localization prediction.
\end{abstract}

\section{(c) 2015 Published by Elsevier Ltd.}

\section{Keywords:}

Protein localization, disease gene identification, gene expression, gene expression, cancer gene

\section{Introduction}

Discovering disease or cancer genes and understanding their underlying pathological mechanisms are the major challenges of biomedical research. In the past decades, many efforts have been devoted to disease gene discovery using either high-throughput techniques [1] or computational disease gene prediction methods [2, 3]. However, these approaches usually report dozens or even hundreds of candidate genes while the experimental validation of many candidates is often an expensive and time-consuming task. To address this issue, many computational candidate gene prioritization algorithms have been developed by exploiting the biomedical knowledge available about the disease of interest and related genes [4]. For example, network information and heterogeneous phenomic and genomic data sources have been used to rank candidate genes [5, 6, 7, 8, 9, 10, 11]. A recent work [4] integrates a plethora of phenomic and genomic data to pinpoint disease genes including disease phenotype similarity derived from the Unified Medical Language System (UMLS) and seven types of gene functional similarities calculated from gene expression, gene ontology, pathway membership, protein sequence, protein domain, protein-protein interaction and regulation pattern, respectively. Their methods thus covered a total of 7,719 diseases and 20,327 genes. However, such studies bring limited insight into the pathological mechanisms due to their generic nature.

Email address: jianjunh@cse.sc.edu (Jianjun Hu)

${ }^{1}$ Corresponding author 
This research proposes a novel approach to identify mislocalized cancer genes that are detected by high-throughput genomic sequencing [1] using the tumor-normal tissue gene expression data.

Eukaryotic cell, as the basic structural and functional unit of eukaryotic living organisms, contains numerous proteins located in different subcellular organelles or compartments, such as nuclei, mitochondria, cytoplasm, and Golgi apparatus (Figure 1). Binder et al. developed a comprehensive database to incorporate all data sources of subcellular localization [12]. Protein translocation is key for a cell to function normally because proteins must be transported to their targeted compartment to exert their functions. The precise trafficking and translocation of cytosolic proteins to their final destinations are crucial for the maintenance of appropriate cell functions and activities. Proteins are typically directed to compartments by short peptide sequences that act as targeting signals. Translocation to the proper compartment allows a protein to form the necessary interactions with its partners and take part in biological network such as signaling and metabolic pathways. If a protein is not translocated to its correct intracellular compartment, either the reaction performed or information carried by the protein does not reach the proper site, causing either inactivation of central reactions or misregulation of signaling cascades, or the mislocalized active protein has harmful effects by acting in the wrong place. This study is focused on identifying potential cancer genes that may be due to their mislocation within the cell.

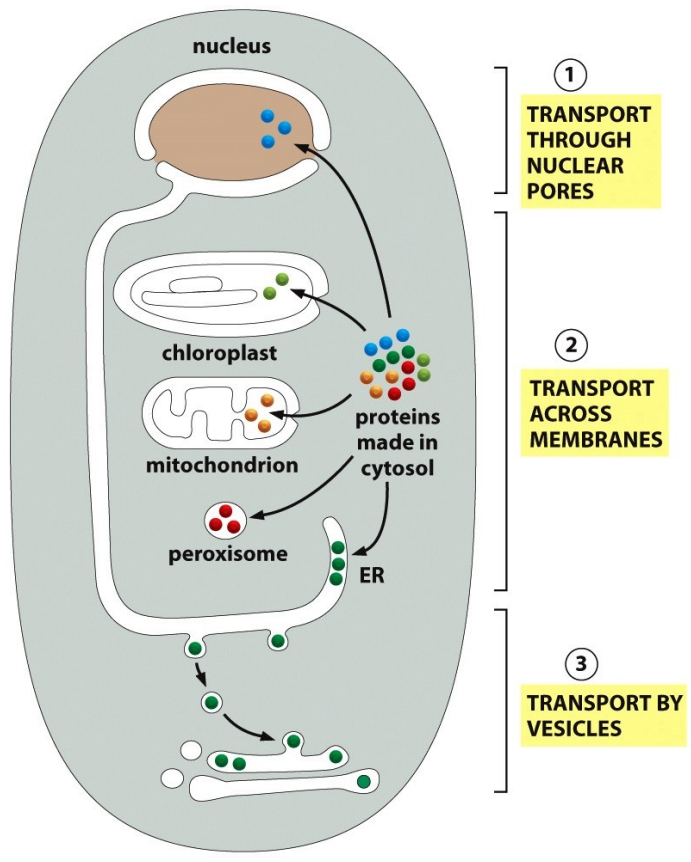

Figure 1. subcellular protein sorting

\section{Subcellular mislocation of proteins}

The protein sequence contains the information regulating protein trafficking [13]. Based on proteins' target destinations, there are two major mechanisms of translocation: post-translational translocation and co-translational translocation $[14,15,16,17]$. Proteins translocating to peroxisomes, mitochondria or the nucleus are called post-translational translocation $[17,18,19,20,21]$. For proteins translocated into the endoplasmic reticulum during synthesis, a process known as co-translational translocation is used [17]. Subcellular localization determines the access of proteins to interacting partners and the post-translational modification machinery and enables the integration of proteins into functional biological network [14].

However, proteins not always go to the correct locations and mislocalized proteins can lead to disease like Alzheimer's disease, kidney stones and cancers [14]. This is because failure to be transported to the correct subcellular compartment can have adverse effects, as protein location is fundamental to the functioning of cells and 
regulatory control in the disease [22]. In [23], three major mechanisms were identified that can lead to protein mislocation: mislocation through alterations of the protein trafficking machinery [24], mislocation through altered protein targeting signals [25] and mislocation through changes in protein interaction or modification [14].

Mislocalized proteins have been shown to be associated with a variety of human diseases such as Swyer syndrome, TRPS, XLAG, speech-language disorder, APECED, Diamond-Blackfan anemia, Laminopathy, Noonan-like syndrome, retinitis pigmentosa, Wilson disease, Tangier disease, Neurodegenerative diseases, ALS and FTLD, and cancers [14]. It is thus interesting to check whether gene expression profiles of tumor-normal tissue pairs can help to identify mislocation related disease/cancer genes.

\section{Protein subcellular location prediction}

To identify subcellular location of proteins, one common experimental technique is immunolabeling when there's suitable antibodies [23]. Anther way is splitting the cell into different subcellular organelles and analyzing the protein content of each by tandem mass spectrometry [26]. However, experimental determination of protein localization is costly and challenging[27,28]. Thus, numerous method and models have been proposed to predict protein subcellular locations, which is essentially a type of protein function annotation or prediction task [29, 30, 31, 32, 33, 34, 35]. Usually, these methods employ machine learning algorithms such as neural network[36], nearest neighbor classifier, Markov models, Bayesian network, logistic regression [37], expert rules, meta-classifiers[38] and the support vector machines[39, 40] on datasets extracted from NCBI Gene Expression Omnibus(GEO) Database, PPI network[41, 42], gene ontology[43] and sequence or structural information to predict protein subcellular localization.

Out of these methods, only a few algorithms have considered the role of gene expression files for localization prediction. Ryngajllogene et al. [44] used the scaled gene expression values for different conditions as features for training SVM predictors. Their results showed that gene expression carries information about the subcellular localization not available in sequences, yielding dramatic benefits for plastid localization prediction, and some notable improvements for other compartments such as tmitochondrion, Golgi apparatus and the plasma membrane. Lee et al. [45] utilized gene expression information for predicting conditional localization. This is the first computational approach for predicting conditional changes in the subcellular location of proteins in a genome-wide manner. They found that functional coherence in the gene expression profiles of protein pairs that are known to interact physically is an indicator of their subcellular location. For each condition and study, each interaction was assigned a functional "coherence" score proportional to both expression level and the correlation between interacting protein pairs. Mondal and $\mathrm{Hu}$ [46] used co-expression network and diffusion kernel logistic regression for protein localization prediction.

In this work, we used NetLoc[47] algorithm that we developed before for prediction of protein localization. Netloc is a network based protein localization prediction algorithm by combining diffusion kernel with logistic regression (KLR) using PPI network including physical PPI network, genetic Protein-Protein Interaction (PPI) network, mixed PPI network and co-expression network[47]. This model showed good performance in protein subcellular localization prediction in the benchmark experiments. More details are described in Methods section.

\section{Cancer/disease gene discovery}

In the past decade, high-throughput methodological approaches such as cancer genome sequencing, RNA sequencing, cancer exome sequencing and functional genomics [2] have led to the identification of multiple cancer-causing genes, genetic alterations and deregulated pathways. Identifying and understanding the underlying mechanisms are the foundation for cancer diagnostics, therapeutics, clinical-trial design and selection of rational combination therapies $[48,49,50,51,52,53,53]$. These hight-throughput experimental approaches plus the computational disease gene prioritization tools can greatly help to pin-down the range of cancer genes for experimental studies.

In a recent genome-wide study, Lawrence et al. [1] analyzed 4,742 human cancers and their matched normaltissue exome sequences accross 21 tumor types. This large-scale genomic analysis identified nearly all known cancer genes in 21 tumor types. Additionally, out of 81 "novel" candidate cancer genes, 33 genes are not previously reported as significantly mutated in cancer. Based on close examination, they found at least 21 novel genes with strong and consistent connections to cancer. With more cancer sequencing, it is expected that a comprehensive catalogue of cancer genes can be discovered to enable physicians to select best therapy fore each patient. However, experimental studies of these newly identified candidate genes are needed to verify and uncover the underlying mechanisms of these cancer-causing genes. 
Lawrence et al.'s study [1] showed the power of tumor-normal pairs of the genome sequence data for discovery of candidate cancer genes, which inspired us to explore how to apply the tumor-normal pairs of gene expression data for identifying the cancer/disease-causing genes that lead to mis-localization of proteins. Actually, Pinto et al. showed that dynamic redistribution of multitudinous proteins to different subcellular locations in response to cellular functional state is a crucial characteristic of cellular function that seems to be at least as important as overall changes in protein abundance [54]. Laurila and Vihinen [55] applied bioinformatics methods to investigate the effects of known disease-related mutations on protein targeting and localization by analyzing over 22,000 missense mutations in more than 1,500 proteins with two complementary prediction approaches. However, many of the localization prediction algorithms that they used are not sensitive enough to capture the subtle sequence mutation to give different localization predictions. Lee et al. [45] proposed an integrative computational framework for mapping conditional location and mislocation of proteins on a proteome-wide scale. They mapped the locations of over 10,000 proteins in normal human brain and in glioma, out of which over 150 have a strong likelihood of mislocation under glioma. Fifteen of these mislocations have been confirmed. The most common type of mislocation occurs between the endoplasmic reticulum and the nucleus.

\section{Problem and our solution}

High-throughput sequencing has almost identified all known and potentially new cancer genes based on tomournormal tissue sequences. It implies that the major task now is on computational or experimental techniques to elucidate the underlying pathologic mechanisms of these cancer-causing genes. In this work, we are interested in discovering cancer genes which impact humans due to their mislocation within the cell. Lee's pipeline was proved successful in discovery of mislocalized proteins in cancer. However the data needed in Lee's pipeline were complex including sequences, chemistry, motifs and gene function ontology $[56,45]$. Considering the data source and lacking of ready-to-use server or software, it is very difficult to use their pipeline to do large scale screening. When predicting conditional localization, we found that in Lee's method the only evidence source that has changed between normal and disease status is gene expression. It is thus natural to develop methods for conditional localization prediction based only on the changing factor - gene expression. Compared to Lee's approach, our approach only depends on gene expression files and gene ontology, which makes it applicable for broader range of cancers. As shown in Figure 3 , our pipeline used gene ontology and gene expression files to predict proteins' subcellular locations in disease/tumor and normal states. Based on predicted locations of two states, we can identify proteins whose subcellular locations change dramatically. The reason that we only use gene expression data as input is that we are trying to predict the condition-specific mislocations. For this problem, the only available changing data is the gene expression data while the PPI data remain the same for both normal and disease states. The reason is that the physical PPI network in the cancers are unknown and cannot be used in the prediction model. If any gene in our candidates is a known cancer gene, it may cause cancer due to its mislocation within the cell. Our work aims to open a way to explore the complex relationship between protein subcellular locations and disease phenotypes.

\section{Material and methods}

The main idea of this study is to predict mislocalized disease genes by predicting subcellular localizations of proteins using gene ontology annotations and gene expression profiles of disease and normal states. The overall procedure of our approach is as follows:

(a) Generate co-expression matrices using MIC[57] package based on tumor-normal gene expression files.

(b) Use KLR logistic regression model[58] to predict the conditional subcellular locations of all proteins in both states

(c) Compare and rank the probability discrepancy of localization of all proteins and get candidates genes that have maximum localization changes.

Our gene co-expression network based localization predictor is based on diffusion kernel logistic regression model (KLR) that we developed using PPI network [47]. As shown in Figure 2, functionally related or interacting proteins tend to be localized to the same subcellular locations. If a disease gene's function is due to its mislocation within the cell, then its co-expression patterns with other related genes will be changed in disease states compared to normal states. 
In the following subsections, we introduce the preparation of PPI network, gene ontology annotation matrix and co-expression matrix needed by KLR. And then diffusion kernel-based logistic regression (KLR) model[58] for subcellular localization prediction is described.

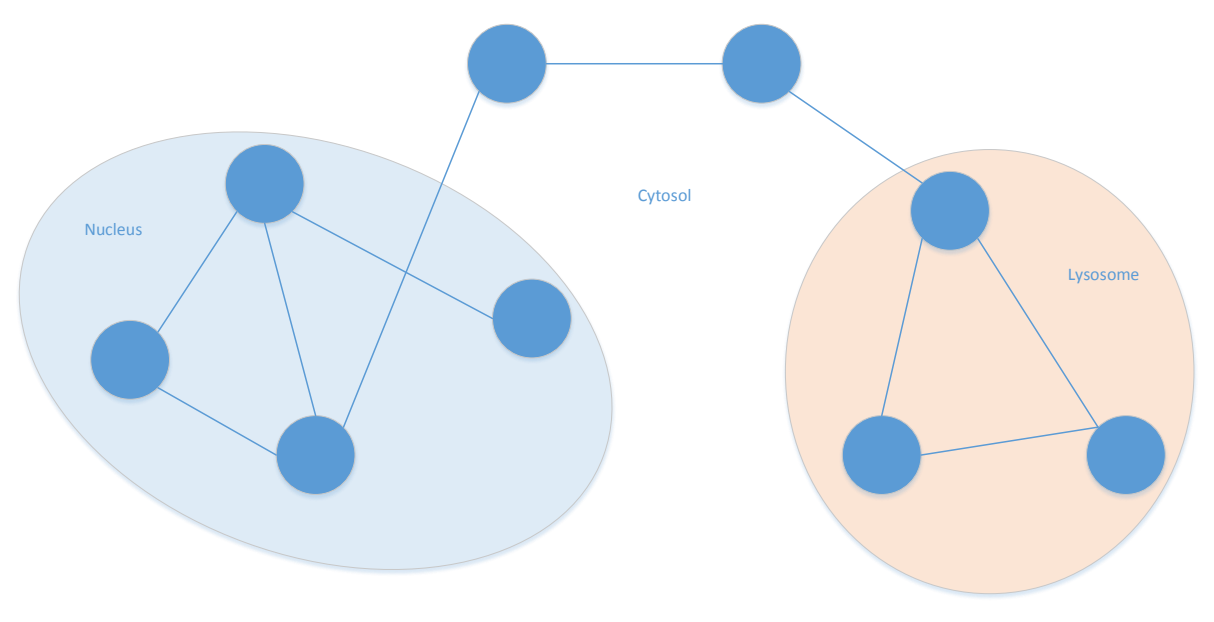

Figure 2. protein-protein interaction function network. Interacting or functionally related proteins tend to be localized to the same subcellular locations

Protein interaction network, gene ontology and protein locations

The input to the KLR model for localization prediction is a PPI network, each node corresponding to a protein. Some of these proteins are annotated with one or more subcellular locations. The KLR model will then learn a model and predict the subcellular locations of unannotated proteins.

To benchmark the KLR model, we generate a protein-protein interaction network based on interactions downloaded from DIP[42] (Released on 2014/04/27) and HPRD[41] (Version number: Relase 9). DIP provides specific datasets for different species and we downloaded Homo sapiens dataset which includes 3651 proteins and 5534 interactions. There are 11269 proteins and 36,398 interactions in HPRD database(Table 1). Combining two databases, we constructed a $12516 \times 12516$ PPI network matrix in which each element $m_{i j}$ represents the interaction status between protein $i$ and $j$ :

$$
m_{i j}= \begin{cases}1 & \text { protein } i \text { and } j \text { have interaction; } \\ 0 & \text { otherwise; }\end{cases}
$$

We obtained subcellular localization annotations of human proteins from GOC website[43]. The datasets contains 44,900 annotated proteins. We then collected the proteins that have at least one of 13 high-subcelluar locations as shown in table 3 as is done by Lee et al.[45] to construct the annotation file used by KLR regression model. We screen the GOC database and 6270 proteins were found appearing in our PPI network(Table 1). Then we got a location annotation file, each row representing a protein's annotation array $A_{p}\left[l_{1}, \ldots, l_{13}\right]$ where:

$$
A_{p}\left[l_{i}\right]= \begin{cases}1 & \text { protein } p \text { was annotated at sub- } \\ 0 & \text { cellular location } l_{i}\end{cases}
$$

Actually, the annotation file is a $6270 \times 13$ annotation matrix. 
Table 1. Source of evidence data: Protein-protein interactions

\begin{tabular}{|c|c|c|}
\hline Data source & Proteins & interactions \\
\hline DIP & 3,685 & 5,570 \\
\hline HPRD & 11,269 & 39,240 \\
\hline Summary & 12,516 & 40,940 \\
\hline
\end{tabular}

\section{Gene expression profiles and co-expression network}

Five types of cancer gene expression profiles and normal human expression profiles were downloaded from the NCBI Gene Expression Omnibus(GEO) Database (table 2). For each of these files, we applied KNN Impute to estimate the missing values. The KNN Impute is downloaded from [59]. All expression profiles were quantile normalized. If multiple probes were mapped to one gene, we use their average values to combine the records. For each dataset we obtained, we split samples into two categories (if applicable): disease samples and normal samples. Then, we generate corresponding co-expression matrices for both normal and disease samples, respectively. Within a co-expression matrix $M$, whose dimension is $N * N$ where $N$ is the number of proteins both covered in the expression profiles and PPI network, element $M_{i j}$ represents the co-expression score of protein $i$ and protein $j$.

Table 2. Gene expression profile for 6 cancers

\begin{tabular}{|c|c|c|}
\hline Cancer & GEO dataset & Samples \\
\hline Acute myeloid leukemia & GSE9476, GSE9476 & 25(disease), 38(normal) \\
\hline Bladder & GSE3167, GSE48276, GSE3239, GSE1595 & 110 (disease), 21(normal) \\
\hline Breast & GSE27567, GSE20437 & 61(disease), 49(normal) \\
\hline Colorectal & GSE21510 & 31 (disease), 25(normal) \\
\hline Diffuse large B-cell lymphoma & GSE14938, GSE43677 & 32(disease), 42(disease) \\
\hline
\end{tabular}

\section{KLR logistic regression model}

In our previous work, we developed diffusion kernel-based logistic regression (KLR) model to predict protein subcellular localization based on the locations of all other proteins within function linkage network [46]. This method has the unique advantage of considering the subcellular location labels of all related proteins[58]. The diffusion kernel provides means to incorporate all neighbors (rather than direct neighbors) of proteins in the network. It also allows each protein annotated with multiple subcellular locations. Multiple protein-protein association network can be combined to enhance the prediction performance. For example, KLR model can incorporate both physical PPI network and co-expression network for localization prediction [47]. KLR model takes a PPI network and subcellular location annotations to calculate the probabilities for query protein to each subcellular location proteins may be localized to. The overall pipeline is shown in Figure3.

The KLR model based protein subcellular localization prediction problem can be formulated as follows[58]. Given a protein-protein interaction network consists of $N$ proteins $\left\{X_{1}, \ldots, X_{N}\right\}, n$ of them have no subcellular location annotations. Our goal is to assign subcellular location GO terms to the $n$ proteins based on the remaining annotated proteins and the PPI network and/or co-expression network. In KLR model, the probability of one protein locating at a subcellular location $\left(X_{i}=1\right)$ is given by:

$$
\operatorname{Pr}\left(X_{i}=1 \mid X_{[N]}, \theta\right)=\frac{1}{1+e^{-\left(\gamma+\delta M_{0}+\eta M_{1}\right)}}
$$

where $\gamma, \delta, \eta$ can be estimated using the Maximum Likelihood Estimation (MLE) and $M_{0}, M_{1}$ is defined as:

$$
\begin{aligned}
& M_{0}(i)=\sum_{j \neq i, x_{j} \text { known }} K(i, j) I\left\{x_{j}=0\right\} \\
& M_{1}(i)=\sum_{j \neq i, x_{j} \text { known }} K(i, j) I\left\{x_{j}=1\right\}
\end{aligned}
$$


where $K(i, j)$ is the kernel function for calculating the similarity distances between two proteins in the network. $I\left\{x_{j}=0\right\}$ is a measure indicating that the interacting protein $j$ does not have the location annotation of interest and $I\left\{x_{j}=1\right\}$ indicates that protein $j$ does localized to the location of interest. Diffusion kernel $K$, to represent the interaction network, is defined using the following equation:

$$
K=e^{\tau L}
$$

Where

$$
L(i, j)= \begin{cases}1 & \text { protein } i \text { interacts with protein } j \\ -d_{i} & \text { protein } i \text { is the same as protein } j \\ 0 & \text { otherwise }\end{cases}
$$

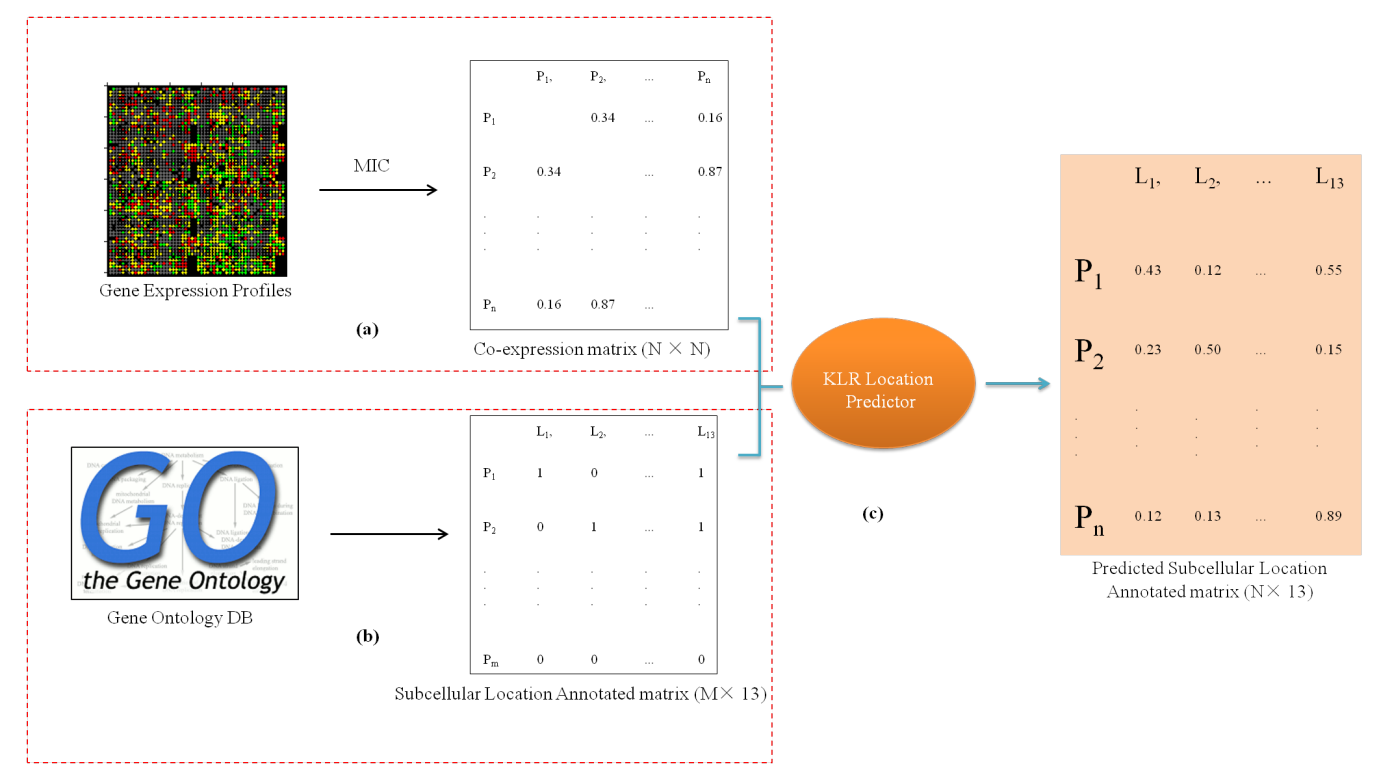

Figure 3. Pipeline of KLR model for subcellular localization prediction. (a) Based on expression values, maximal information coeeficient was calculated of two proteins. Then we got a co-expression matrix $(N \times N)$ which was considered as a condition-based PPI network. (b) In gene ontology database, we extracted subcellular ontology information of any possible protein appeared in gene expression profile to generate the subcellular location annotated matrix $(M \times 13)$. (c) KLR predictor took two matrix as input to predict the probabilities on 13 subcellular locations of each protein appeared in gene expression file. The result was a $N \times 13$ subcellular location annotated matrix.

\section{Results}

\section{Performance evaluation of KLR based protein localization prediction}

The main idea of the KLR localization prediction algorithm is that if two proteins interact (functionally, physically, or genetically) with each other, then they are more likely to be located at the same subcellular location. To check this hypothesis and evaluate the prediction performance of our network-based protein localization algorithm, we conducted a validation experiment using PPI network $[42,41]$. In this experiment, we didn't use co-expression network to make sure that prediction results would not be affected by different co-expression states. The PPI network was retrieved from DIP and HPRD as described in Methods Section (See Table 1). It has 12516 proteins and 40940 interactions. Out of these proteins, 6270 of them have localization annotation belong to 13 subcellular locations (Table 3).

We applied leave-one-out cross-validation approach to benchmark the KLR prediction performance over all proteins with subcellular location annotation. For each gene $g$ with subcellular location annotation, the leave-one-out cross-validation is done as below: 
Table 3. Subcellular locations

\begin{tabular}{|c|c|c|}
\hline \multicolumn{2}{|c}{ Table 3. Subcellular locations } \\
\hline Source & Gene ontology ID & Location \\
\hline \multirow{5}{*}{ GO:0005938 } & cell cortex \\
\cline { 2 - 3 } & GO:0005829 & cytosol \\
\cline { 2 - 3 } & GO:0015629 & actin cytoskeleton \\
\cline { 2 - 3 } & GO:0005794 & Golgi apparatus \\
\cline { 2 - 3 } & GO:0005783 & endoplasmic reticulum \\
\cline { 2 - 3 } & GO:0005773 & vacuole \\
\cline { 2 - 3 } & GO:0005730 & nucleolus \\
\cline { 2 - 3 } & GO:0005777 & peroxisome \\
\cline { 2 - 3 } & GO:0005739 & mitochondrion \\
\cline { 2 - 3 } & GO:0005764 & lysosome \\
\cline { 2 - 3 } & GO:0005813 & centrosome \\
\cline { 2 - 3 } & GO:0005634 & nucleus \\
\cline { 2 - 3 } & GO:0005886 ma membrane \\
\hline
\end{tabular}

1. Delete annotation entry of the target gene $g$ in the annotation file to get a new annotation file. Use KLR model to predict the subcelluar location of gene $g$ with new annotation file;

2. For KLR's prediction result, we pick top 3 subcelluar locations with highest probabilities as the predicted subcellular locations. Then we compare these 3 locations with the ground truth annotated locations. If an annotated location is in the predicted results, we mark this prediction as a correct prediction. Otherwise, we mark it as a wrong prediction.

Table 4. Known subcelluar locations of human proteins

\begin{tabular}{|c|c|}
\hline Data source & GOC \\
\hline Proteins annotated with subcellular locations & 15021 \\
\hline Annonated proteins found in PPI network & 6270 \\
\hline
\end{tabular}

Totally, we evaluated all the 6270 genes with subcellular localization annotations (Table 4), out of which 5,242 genes' subcellular locations were predicted correctly (table 5). The accuracy is $83.62 \%$. Our verification experiment indicated that KLR model indeed can predict subcelluar locations with high accuracy based on PPI network and protein subcellular localization annotation. However, due to the fact that many proteins (more than one third) are annotated with multiple subcellular localizations [60], the accuracy is not perfect yet.

To benchmark the capability of KLR for predicting the condition specific localization, it is ideal to use different co-expression network with ground truth locations in either condition. To do that, we evaluate our method using dataset provided in Lee's paper, the result was reported in section below.

\section{Maximal information coefficient V.S. Pearson coefficient}

Our approach was trying to find out cancer genes that cause disorders due to their mislocation within the cell. Such mislocalizations should be reflected by their co-expression patterns with other genes involved in the same function or pathway. How to define the co-expression relationship of genes is thus critical to our approach. Previous work on coexpression network usually use Pearson correlation coefficient with some threshold values to determine the network [61]. Pearson correlation coefficient is a common tool used to evaluate expression coherence of proteins. However, the normalized expression level of two proteins does not need to follow linear relationship to be functionally correlated. Coherent non-linear relationship between genes also means functional relationships. In this case, Maximal information coefficient (MIC)[57], which capture non-linear dependency between two variables may be more appropriate to capture the linear or nonlinear function relationships.

Figure 4 shows the co-expression relationships of several pairs of genes with different MIC scores along with their Pearson correlation scores. From subfigure (d) in which MIC was 0.93 and Pearson coefficient was 0.65 , we could 


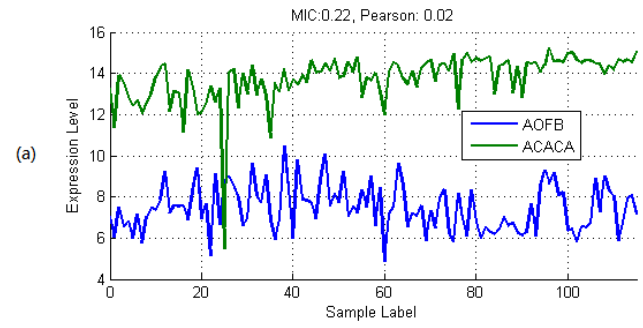

(b)
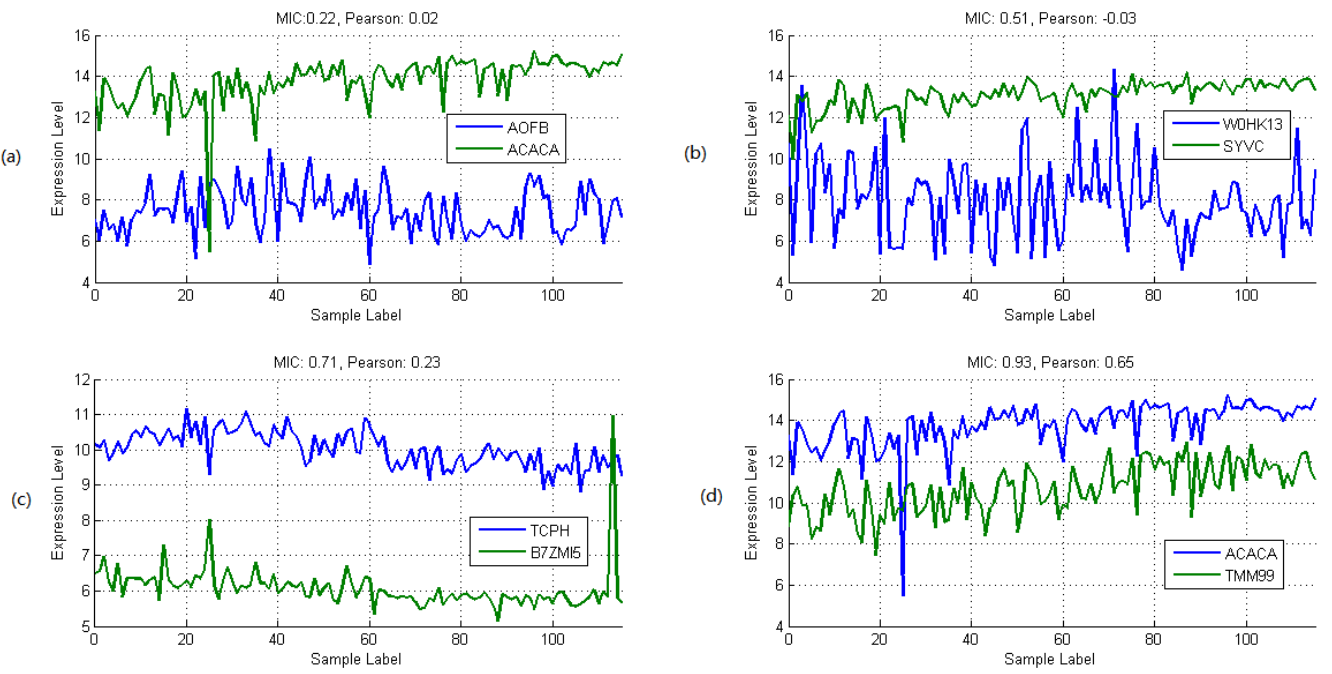

Figure 4. Comparison of MIC and Pearson Coefficients for Co-expressed proteins. (a) Co-expression of protein AOFB and protein ACACA. The MIC was 0.22 and the Pearson coefficient was 0.02 based on plotted expression values. (b) Co-expression of protein W0HK13 and protein SYVC. The MIC was 0.51 and the Pearson coefficient was -0.03 based on plotted expression values. (c) Co-expression of protein TCPH and protein B7ZMI5. The MIC was 0.71 and the Pearson coefficient was 0.23 based on plotted expression values. (d) Co-expression of protein ACACA and protein TMM99. The MIC was 0.93 and the Pearson coefficient was 0.65 based on plotted expression values.

find that protein ACACA and TMM99 actually has a very strong co-expression relationship. And MIC detected this kind of relationship. MIC was proposed by Reshef et al. aiming at capturing a wide range of non-linear associations between two random variables. In order to check whether MIC can better capture functionally related proteins and thus give us better protein localization prediction performance, we applied KLR based localization algorithm to predict the subcellular locations using the co-expression matrix calculated from these two types of correlation coefficients. We used GDS3716 [62] from GEO database to calculate the co-expression matrices.

The procedure of the leave-one-out evaluation experiment is as below:

1. Calculate the co-expression matrices with both MIC and Pearson Coefficient and train the KLR models.

2. Predict the left-out gene's subcellular locations with trained KLR model using the remaining annotated proteins.

3. Compare predictions with real annotated subcellular locations.

4. Calculate accuracy based on all leave-one-out prediction results.

In table 5, we showed the prediction results based on leave-one-out cross-validation evaluation over 6270 genes. Here the top 1 accuracy means for each left-out test gene, we calculate the percentage of the number of cases that the best predicted location with highest probability score matches the true answer. The top 2 accuracy means for each left-out test gene, we calculate the percentage of the number of cases that one of the two best predicted location with highest probability scores matches the true answer. Top 3 accuracy is also defined similarly. For the top 1 accuracy, the MIC based KLR achieved $4.71 \%$ higher prediction accuracy. For the top 3 accuracy, it achieves $6.49 \%$ higher in terms of prediction accuracy, which is a significant improvement. Our experiment results showed that compared with Pearson coefficients, MIC based KLR in general had higher prediction accuracy to predicting the subcellular locations due to their detection of nonlinearly related proteins in terms of gene expression levels (Table 5).

\section{Rediscovery of mislocalized proteins in glioma}

In the recent work of Lee [45], a proteome-wide method was proposed to predict mislocalized proteins under glioma tumor. They used sequence, chemical properties, motifs, and function information of proteins as the basic features. Condition-dependent dynamic network features were generated by assigning different weights to each neighbor 
Table 5. Comparing MIC and Pearson Coefficient

\begin{tabular}{|c|c|c|c|}
\hline \multirow{2}{*}{ Method } & \multicolumn{3}{|c|}{ Prediction accuracy with different No. of candidate } \\
\cline { 2 - 4 } & top 1 & top 2 & top 3 \\
\hline MIC & $41.09 \%$ & $63.74 \%$ & $85.37 \%$ \\
\hline Pearson & $36.39 \%$ & $60.41 \%$ & $78.88 \%$ \\
\hline Difference & $4.71 \%$ & $3.33 \%$ & $6.49 \%$ \\
\hline
\end{tabular}

of a protein, depending on their similarity in gene expression profiles. Based on the basic features and dynamic network features, they computed a CLM score for each protein, listing the quantitative probability that the protein is located in each location under each condition. Mislocations are identified by calculating differences in degrees of probabilities across conditions. Their prediction result showed that their model can successfully identify potential mislocation proteins under tumor conditions. However, their approach used many data resources besides gene expression files, which might not be available for some proteins. Since the condition-dependent information is only contained in the gene expression data, we wanted to test our model on the same gene expression data to see if we can rediscover mislocalized proteins under glioma. We used GEO dataset used by Lee's team which contained normal, low and high states $[63,64,65,66,67,68]$. In order to find possible mislocalized proteins, firstly, our pipeline was used to predict location probabilities of 13 subcellular locations of each protein for each status. For each protein, we selected the location with highest probability as its predicted location in that status. After we got a protein's location probabilities on normal, low and high statuses, we just picked up proteins with different locations under normal and disease statuses. Totally, we found 230 proteins predicted as mislocalized proteins among 11500 proteins. And for 157 proteins predicted as potentially mislocalized under glioma in Lee's paper, we rediscovered 31 proteins of them in our 230 predicted candidates having same mislocation activity. Among rediscovered 31 proteins, TBX19 was experimentally verified mislocation from endoplasmic reticulum to nucleus within glioma cell in in Lee's paper. The result is shown in table 6.

Table 6. List of rediscovered mislocalized proteins in glioma

\begin{tabular}{|l|l|}
\hline \multirow{3}{*}{ Mislocalized proteins under glioma } & PSPN, TMEM132A, ARF5, TIA1, TIMM8A, OBP2A, CIC, OLFM2, \\
CPSF3L, DUSP14, PPM1B, SEC13, SSBP3, PEX13, THPO, RCAN2, \\
TTN, WNT6, TBX19, DAP, GEM, ATIC, DCPS, PROC, PAX1, SSB, \\
TYMS, CCDC116, FTL, CBLL1, PLK3,
\end{tabular}

\section{Case studies: discovery of possible mislocalized cancer proteins across several types of cancers}

Firstly, we test our pipeline on 5 types of cancers (Acute myeloid leukemia, Bladder, Breast, Colorectal and Diffuse large B-cell lymphoma) reported in [1]. From GEO, we downloaded gene expression files of normal state and cancer state tissues for each kind of cancers (table 2). Subcellular localization probabilities were calculated for all proteins and for both normal and tumor states. Then we got conditional localization probability changes of all proteins in gene expression files. Next, for each cancer type we sorted the candidate cancer proteins reported in [1] by the localization probability change for each subcellular location. We reported the proteins with either at least $10 \%$ probability change or ranked within top 4 proteins with at least $5 \%$ probability change. Probability change $\Delta p$ was calculated from $\Delta p=\frac{p_{i, d i s e a s e}-p_{i, \text { normal }}}{p_{\text {innormal }}}$ where $p_{i, \text { disease }}, p_{i, \text { normal }}$ are the protein probabilities of locating on subcellular location $i$ in disease and normal cell, respectively. If $\Delta p$ was a relatively large negative value, it indicated that the protein may be mislocated from subcellular location $i$ in disease state. And if $\Delta p$ was a relatively large positive value, it indicated that the protein may be mislocated to the subcellular location $i$ in disease state. We observed that in most cases, the cancer genes not selected in our method usually had much lower probability change with a $p$-value of less than $0.002 \%$ for $t$ test of all $\Delta p$ on a subcellular location $i$. So the simple threshold approach adopted here to selecting mislocated proteins is sufficient to distinguish the potential mislocalized cancer genes from other genes. Table 7 shows the results. 
Table 7. Potential mislocalized cancer gene candidates identified by KLR

\begin{tabular}{|c|c|c|}
\hline Cancer type & $\begin{array}{l}\text { Mislocalized protein candidates and } \\
\text { subcellular places mislocalized to }\end{array}$ & Missing protein candidates and location \\
\hline $\begin{array}{l}\text { Acute } \\
\text { myeloid } \\
\text { leukemia }\end{array}$ & $\begin{array}{l}\text { STAG2 (Nucleus[GO:0005634], Endo- } \\
\text { plasmic reticulum[GO:0005783]) }\end{array}$ & $\begin{array}{lll}\text { DNMT3A } & \text { (Golgi } & \text { appara- } \\
\text { tus[GO:0005794]) } & & \end{array}$ \\
\hline Bladder & $\begin{array}{l}\text { DDX5(Nucleus[GO:0005634]) } \\
\text { ELF3 (Mitochondrion[GO:0005739]) }\end{array}$ & 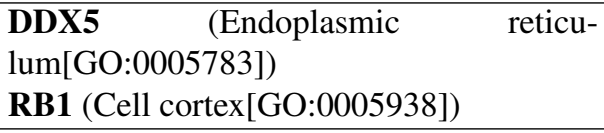 \\
\hline Breast Cancer & $\begin{array}{l}\text { ERBB3 (Golgi apparatus[GO:0005794]) } \\
\text { ERBB2 (Golgi apparatus[GO:0005794]) }\end{array}$ & $\begin{array}{l}\text { ERBB3 (Nucleolus[GO:0005730], } \\
\text { ium[GO:0005929]) } \\
\text { ERBB2 (Nucleolus[GO:0005730], } \\
\text { ium[GO:0005929]) }\end{array}$ \\
\hline Colorectal & PIK3CA (Cytosol[GO:0005829]) & $\begin{array}{l}\text { PIK3CA (Golgi apparatus[GO:0005794]) } \\
\text { GOT1 (Peroxisome[GO:0005777], Endo- } \\
\text { plasmic reticulum[GO:0005783]) }\end{array}$ \\
\hline $\begin{array}{l}\text { Diffuse large } \\
\text { B-cell } \\
\text { lymphoma }\end{array}$ & $\begin{array}{l}\text { CD70 (plasma membrane[GO:0005886]) } \\
\text { CREBBP (Golgi } \\
\text { tus[GO:0005794]) } \\
\text { MAP2K1 (Cytosol[GO:0005829]) }\end{array}$ & $\begin{array}{l}\text { CD70 (Nucleus[GO:0005634]) } \\
\text { CREBBP (Mitochondrion[GO:0005739]) } \\
\text { B2M (Golgi apparatus[GO:0005794]) } \\
\text { EZH2 (Centrosome[GO:0005813]) }\end{array}$ \\
\hline
\end{tabular}

\section{Acute myeloid leukemia}

In Acute myeloid leukemia, we used 25 samples in tumour state and 38 samples in normal state as our analysis data. They were all from hematopoietic cells . Our pipeline identified two genes DNMT3A and STAG2 with high localization change that are reported as cancer genes in [1]. DNMT3A, in our model, was predicted mislocating from Golgi apparatus in disease cell $(2.96 \%)$ comparing with normal cells(6.29\%). Another covered candidate gene is STAG2, which was predicted mislocating to two locations: Nucleus where had $41.29 \%$ probability in normal and had probability $64.45 \%$ in disease and Endoplasmic reticulum where had 5.52\% probability in normal and had probability $12.12 \%$

\section{Bladder tumor}

Our results used 9 normal bladder tissue samples and more than 110 disease bladder tissue samples. The cancer gene DDX5 was predicted to have a $36.19 \%$ probability of being localized to Nucleus in normal tissues. The predicted probability of being localized to Neucleus in disease tissues was $52.25 \%$ with an increase of more than $40 \%$. At the same time, DDX5 had $8.60 \%$ probability in normal and $3.80 \%$ in disease localizing on Endoplasmic reticulum with an decrease of more than $55 \%$. So based on this probabilities changes, we can make a speculation that in bladder tumor cell DDX5 mislocalized from Endoplasmic reticulum to neucleus. Tumor suppressor gene RB1 was predicted with a high probability change in its subcellular location from cell cortex to other locations $(3.39 \%$ in normal, $0.98 \%$ in disease). Recently a study [69] showed that in breast cancer mutated RB1 will lead to their localization to nucleus. We also observed in our prediction the increase of probability localization in nucleus $(48.23 \%$ in normal, $57.27 \%$ in disease). Our predicted localization change of RB1 maybe caused by its mutation. Also protein ELF3 was predicted mislocating to Mitochondrion where its location probability changing from $8.16 \%$ in normal state to $15.31 \%$ in cancer state.

\section{Breast Cancer}

After analyzing 61 disease samples and 49 normal samples, We obtained several candidate proteins that probably mislocate under cancer states. At subcellular location Cilium, protein ERBB2's predicted localization probability 
decreased from $63.68 \%$ (normal) to $55.87 \%$ (disease). Also its probability localization on Nucleolus had a decreasing from $6.41 \%$ (normal) to $1.99 \%$ (disease). And at the same time, ERBB2's probability localization on Golgi apparatus had an increasing from $8.65 \%$ (normal) to $11.37 \%$ (disease). These observations could indicate that some ERBB2 proteins in breast cancer cells mislocalized from Cilium and Nucleolus to Golgi apparatus. Protein ERBB3 had similar mislocation activity from from Cilium $(52.77 \%$ in normal, $44.78 \%$ in disease) and Nucleolus $(9.60 \%$ in normal, $3.05 \%$ in disease) to Golgi apparatus ( $8.21 \%$ in normal, $10.75 \%$ in disease).

\section{Colorectal tumor}

For colorectal tumor, we had 31 tumor samples and 25 normal samples. Protein PIK3CA was observed in our prediction mislocating from Golgi apparatus $(11.17 \%$ in normal, $4.81 \%$ in disease) to Cytosol $(27.74 \%$ in normal, $45.84 \%$ in disease). Protein GOT1 was predicted mislocating from Peroxisome where its location probability decreased from $5.09 \%$ to $0.84 \%$ and from Endoplasmic reticulum where its location probability decreased from $10.95 \%$ to $3.99 \%$.

\section{Diffuse large B-cell lymphoma}

In Diffuse large B-cell lymphoma test, we used 42 normal samples and 32 disease samples. Protein CD70 was predicted missing from Nucleus (59.45\% in normal, $35.96 \%$ in disease) and at the same time its probability in Plasma membrane increased from $6.41 \%$ to $39.84 \%$. So we speculated that some CD70 proteins may mislocate to Plasma membrane from Nucleus in cancer cells. Protein CREBBP was also predicted having mislocation activity that from Mitochondrion to Golgi apparatus. Its predicted probability in Mitochondrion decreased from $25.64 \%$ in normal to $6.26 \%$ in disease and the probability in Golgi apparatus increased from $3.95 \%$ to $7.62 \%$. Besides, protein MAP2K1 was observed mislocating to Cytosol (38.22\% in normal, 66.17\% in disease). Protein B2M and protein EZH2 were predicted mislocating from Golgi apparatus (12.90\% in normal, 5.90\% in disease) and Centrosome $(23.92 \%$ in normal, $6.90 \%$ in disease), respectively.

\section{Conclusions}

In this paper, we have developed an approach for discovering mis-location related disease/cancer genes based on aberrant gene expression data and diffusion kernel based logistic regression for subcellular localization prediction. Our approach is complementary to high-throughput genomic sequencing approaches for cancer gene detection by providing the means for understanding the pathological mechanisms. The experiments showed that our approach has identified several interesting cancer genes reported by genomic study, by means of which the cancer may be related to their mis-localization within the cell.

\section{Acknowledgement}

This work was supported by the National Science Foundation Career Award (Grant BIO-DBI-0845381). The funder had no role in study design, data collection and analysis, decision to publish, or preparation of the manuscript.

\section{References}

[1] M. S. Lawrence, P. Stojanov, C. H. Mermel, J. T. Robinson, L. A. Garraway, T. R. Golub, M. Meyerson, S. B. Gabriel, E. S. Lander, G. Getz, Discovery and saturation analysis of cancer genes across 21 tumour types, Nature.

[2] P. M. Lizardi, M. Forloni, N. Wajapeyee, Genome-wide approaches for cancer gene discovery, Trends Biotechnol. 29 (11) (2011) 558-568.

[3] N. Gill, S. Singh, T. C. Aseri, Computational disease gene prioritization: an appraisal, J. Comput. Biol. 21 (6) (2014) 456-465.

[4] R. Jiang, M. Wu, L. Li, Pinpointing disease genes through phenomic and genomic data fusion, BMC Genomics 16 Suppl 2 (2015) S3.

[5] D. Nitsch, J. P. Goncalves, F. Ojeda, B. de Moor, Y. Moreau, Candidate gene prioritization by network analysis of differential expression using machine learning approaches, BMC Bioinformatics 11 (2010) 460.

[6] B. Xie, G. Agam, S. Balasubramanian, J. Xu, T. C. Gilliam, N. Maltsev, D. Bornigen, Disease gene prioritization using network and feature, J. Comput. Biol. 22 (4) (2015) 313-323. 
[7] Z. Q. Zhao, G. S. Han, Z. G. Yu, J. Li, Laplacian normalization and random walk on heterogeneous networks for disease-gene prioritization, Comput Biol Chem 57 (2015) 21-28.

[8] E. Guney, B. Oliva, Analysis of the robustness of network-based disease-gene prioritization methods reveals redundancy in the human interactome and functional diversity of disease-genes, PLoS ONE 9 (4) (2014) e94686.

[9] S. Jiao, Q. Chu, Y. Wang, Z. Xie, S. Hou, A. Liu, H. Wu, L. Liu, F. Geng, C. Wang, C. Qin, R. Tan, X. Huang, S. Tan, M. Wu, X. Xu, X. Liu, $\mathrm{Y}$. Yu, Y. Zhang, Identification of the causative gene for Simmental arachnomelia syndrome using a network-based disease gene prioritization approach, PLoS ONE 8 (5) (2013) e64468.

[10] J. P. Goncalves, A. P. Francisco, Y. Moreau, S. C. Madeira, Interactogeneous: disease gene prioritization using heterogeneous networks and full topology scores, PLoS ONE 7 (11) (2012) e49634.

[11] E. Guney, B. Oliva, Exploiting protein-protein interaction networks for genome-wide disease-gene prioritization, PLoS ONE 7 (9) (2012) e43557.

[12] J. X. Binder, S. Pletscher-Frankild, K. Tsafou, C. Stolte, S. I. O’Donoghue, R. Schneider, L. J. Jensen, COMPARTMENTS: unification and visualization of protein subcellular localization evidence, Database (Oxford) 2014 (2014) bau012.

[13] S. Negi, S. Pandey, S. M. Srinivasan, A. Mohammed, C. Guda, LocSigDB: a database of protein localization signals, Database (Oxford) 2015.

[14] M.-C. Hung, W. Link, Protein localization in disease and therapy, Journal of cell science 124 (20) (2011) 3381-3392.

[15] D. J. Schnell, D. N. Hebert, Protein translocons: multifunctional mediators of protein translocation across membranes, Cell 112 (4) (2003) 491-505.

[16] T. A. Rapoport, Protein translocation across the eukaryotic endoplasmic reticulum and bacterial plasma membranes, Nature 450 (7170) (2007) 663-669.

[17] W. Wickner, R. Schekman, Protein translocation across biological membranes, science 310 (5753) (2005) 1452-1456.

[18] O. Schmidt, N. Pfanner, C. Meisinger, Mitochondrial protein import: from proteomics to functional mechanisms, Nature reviews Molecular cell biology 11 (9) (2010) 655-667.

[19] M. Suntharalingam, S. R. Wente, Peering through the pore: nuclear pore complex structure, assembly, and function, Developmental cell 4 (6) (2003) 775-789.

[20] L. J. Terry, E. B. Shows, S. R. Wente, Crossing the nuclear envelope: hierarchical regulation of nucleocytoplasmic transport, Science 318 (5855) (2007) 1412-1416.

[21] J. Wolf, W. Schliebs, R. Erdmann, Peroxisomes as dynamic organelles: peroxisomal matrix protein import, FEBS journal 277 (16) (2010) 3268-3278.

[22] K. Munkres, K. Benveniste, J. Gorski, C. Zuiches, Genetically induced subcellular mislocation of neurospora mitochondrial malate dehydrogenase, Proceedings of the National Academy of Sciences 67 (1) (1970) 263-270.

[23] M. Uhlen, P. Oksvold, L. Fagerberg, E. Lundberg, K. Jonasson, M. Forsberg, M. Zwahlen, C. Kampf, K. Wester, S. Hober, et al., Towards a knowledge-based human protein atlas, Nature biotechnology 28 (12) (2010) 1248-1250.

[24] M. N. Chahine, G. N. Pierce, Therapeutic targeting of nuclear protein import in pathological cell conditions, Pharmacological reviews 61 (3) (2009) 358-372.

[25] K. Laurila, M. Vihinen, Prediction of disease-related mutations affecting protein localization, BMC genomics 10 (1) (2009) 122.

[26] A. Gilchrist, C. E. Au, J. Hiding, A. W. Bell, J. Fernandez-Rodriguez, S. Lesimple, H. Nagaya, L. Roy, S. J. Gosline, M. Hallett, et al., Quantitative proteomics analysis of the secretory pathway, Cell 127 (6) (2006) 1265-1281.

[27] A. Kumar, S. Agarwal, J. A. Heyman, S. Matson, M. Heidtman, S. Piccirillo, L. Umansky, A. Drawid, R. Jansen, Y. Liu, et al., Subcellular localization of the yeast proteome, Genes \& development 16 (6) (2002) 707-719.

[28] W.-K. Huh, J. V. Falvo, L. C. Gerke, A. S. Carroll, R. W. Howson, J. S. Weissman, E. K. O’Shea, Global analysis of protein localization in budding yeast, Nature 425 (6959) (2003) 686-691.

[29] C. A. Wilson, J. Kreychman, M. Gerstein, Assessing annotation transfer for genomics: quantifying the relations between protein sequence, structure and function through traditional and probabilistic scores, Journal of molecular biology 297 (1) (2000) $233-249$.

[30] R. Mott, J. Schultz, P. Bork, C. P. Ponting, Predicting protein cellular localization using a domain projection method, Genome research 12 (8) (2002) 1168-1174

[31] R. Casadio, P. L. Martelli, A. Pierleoni, The prediction of protein subcellular localization from sequence: a shortcut to functional genome annotation, Briefings in functional genomics \& proteomics 7 (1) (2008) 63-73.

[32] O. Emanuelsson, S. Brunak, G. von Heijne, H. Nielsen, Locating proteins in the cell using targetp, signalp and related tools, Nature protocols 2 (4) (2007) 953-971.

[33] J. L. Gardy, F. S. Brinkman, Methods for predicting bacterial protein subcellular localization, Nature Reviews Microbiology 4 (1) (2006) 741-751.

[34] K. Lee, D.-W. Kim, D. Na, K. H. Lee, D. Lee, Plpd: reliable protein localization prediction from imbalanced and overlapped datasets, Nucleic acids research 34 (17) (2006) 4655-4666.

[35] S. Briesemeister, J. Rahnenfuhrer, O. Kohlbacher, Going from where to why-interpretable prediction of protein subcellular localization, Bioinformatics 26 (9) (2010) 1232-1238.

[36] C. Mooney, Y. H. Wang, G. Pollastri, SCLpred: protein subcellular localization prediction by N-to-1 neural networks, Bioinformatics 27 (20) (2011) 2812-2819.

[37] S. Wan, M. W. Mak, S. Y. Kung, mPLR-Loc: an adaptive decision multi-label classifier based on penalized logistic regression for protein subcellular localization prediction, Anal. Biochem. 473 (2015) 14-27.

[38] K. Laurila, M. Vihinen, PROlocalizer: integrated web service for protein subcellular localization prediction, Amino Acids 40 (3) (2011) 975-980.

[39] L. Zhang, B. Liao, D. Li, W. Zhu, A novel representation for apoptosis protein subcellular localization prediction using support vector machine, J. Theor. Biol. 259 (2) (2009) 361-365.

[40] Z. H. Zhang, Z. H. Wang, Z. R. Zhang, Y. X. Wang, A novel method for apoptosis protein subcellular localization prediction combining 
encoding based on grouped weight and support vector machine, FEBS Lett. 580 (26) (2006) 6169-6174.

[41] T. S. Keshava Prasad, R. Goel, K. Kandasamy, S. Keerthikumar, S. Kumar, S. Mathivanan, D. Telikicherla, R. Raju, B. Shafreen, A. Venugopal, L. Balakrishnan, A. Marimuthu, S. Banerjee, D. S. Somanathan, A. Sebastian, S. Rani, S. Ray, C. J. Harrys Kishore, S. Kanth, M. Ahmed, M. K. Kashyap, R. Mohmood, Y. L. Ramachandra, V. Krishna, B. A. Rahiman, S. Mohan, P. Ranganathan, S. Ramabadran, R. Chaerkady, A. Pandey, Human Protein Reference Database-2009 update., Nucleic acids research 37 (Database issue) (2009) D767-72.

[42] L. Salwinski, C. S. Miller, A. J. Smith, F. K. Pettit, J. U. Bowie, D. Eisenberg, The Database of Interacting Proteins: 2004 update., Nucleic acids research 32 (Database issue) (2004) D449-51.

[43] M. Ashburner, C. Ball, J. Blake, D. Botstein, Gene Ontology: tool for the unification of biology, Nature ... 25 (may) (2000) 25-29.

[44] M. Ryngajllo, L. Childs, M. Lohse, F. M. Giorgi, A. Lude, J. Selbig, B. Usadel, SLocX: Predicting Subcellular Localization of Arabidopsis Proteins Leveraging Gene Expression Data, Front Plant Sci 2 (2011) 43.

[45] K. Lee, K. Byun, W. Hong, H.-Y. Chuang, C.-G. Pack, E. Bayarsaikhan, S. H. Paek, H. Kim, H. Y. Shin, T. Ideker, et al., Proteome-wide discovery of mislocated proteins in cancer, Genome research 23 (8) (2013) 1283-1294.

[46] A. Mondal, J. Hu, Network based prediction of protein localisation using diffusion kernel, International Journal of Data Mining and Bioinformatics 9 (4) (2014) 386-400.

[47] A. M. Mondal, J. Hu, NetLoc: Network based protein localization prediction using protein-protein interaction and co-expression networks, in: Bioinformatics and Biomedicine (BIBM), 2010 IEEE International Conference on, IEEE, 2010, pp. 142-148.

[48] R. E. McIntyre, L. van der Weyden, D. J. Adams, Cancer gene discovery in the mouse, Curr. Opin. Genet. Dev. 22 (1) (2012) 14-20.

[49] T. Nawy, Cancer gene discovery goes viral, Nat. Methods 9 (9) (2012) 868.

[50] J. de Jong, J. de Ridder, L. van der Weyden, N. Sun, M. van Uitert, A. Berns, M. van Lohuizen, J. Jonkers, D. J. Adams, L. F. Wessels, Computational identification of insertional mutagenesis targets for cancer gene discovery, Nucleic Acids Res. 39 (15) (2011) e105.

[51] L. Zender, A. Villanueva, V. Tovar, D. Sia, D. Y. Chiang, J. M. Llovet, Cancer gene discovery in hepatocellular carcinoma, J. Hepatol. 52 (6) (2010) 921-929.

[52] J. Mattison, L. van der Weyden, T. Hubbard, D. J. Adams, Cancer gene discovery in mouse and man, Biochim. Biophys. Acta 1796 (2) (2009) $140-161$

[53] S. A. Tomlins, A. M. Chinnaiyan, Of mice and men: cancer gene discovery using comparative oncogenomics, Cancer Cell 10 (1) (2006) 2-4.

[54] G. Pinto, A. A. Alhaiek, J. Godovac-Zimmermann, Proteomics reveals the importance of the dynamic redistribution of the subcellular location of proteins in breast cancer cells, Expert Rev Proteomics 12 (1) (2015) 61-74

[55] K. Laurila, M. Vihinen, Prediction of disease-related mutations affecting protein localization, BMC Genomics 10 (2009) 122.

[56] K. Lee, H.-Y. Chuang, A. Beyer, M.-K. Sung, W.-K. Huh, B. Lee, T. Ideker, Protein networks markedly improve prediction of subcellular localization in multiple eukaryotic species, Nucleic acids research 36 (20) (2008) e136-e136.

[57] D. N. Reshef, Y. a. Reshef, H. K. Finucane, S. R. Grossman, G. McVean, P. J. Turnbaugh, E. S. Lander, M. Mitzenmacher, P. C. Sabeti, Detecting novel associations in large data sets., Science (New York, N.Y.) 334 (6062) (2011) 1518-24.

[58] H. Lee, Z. Tu, M. Deng, Diffusion kernel-based logistic regression models for protein function prediction, Omics: a journal of integrative biology 10 (1).

[59] O. Troyanskaya, M. Cantor, G. Sherlock, P. Brown, T. Hastie, R. Tibshirani, D. Botstein, R. B. Altman, Missing value estimation methods for DNA microarrays., Bioinformatics (Oxford, England) 17 (6) (2001) 520-5.

[60] S. Zhang, X. Xia, J. Shen, Y. Zhou, Z. Sun, DBMLoc: a Database of proteins with multiple subcellular localizations, BMC Bioinformatics 9 (2008) 127.

[61] P. Langfelder, S. Horvath, WGCNA: an R package for weighted correlation network analysis, BMC Bioinformatics 9 (2008) 559.

[62] K. Graham, A. de Las Morenas, A. Tripathi, C. King, M. Kavanah, J. Mendez, M. Stone, J. Slama, M. Miller, G. Antoine, et al., Gene expression in histologically normal epithelium from breast cancer patients and from cancer-free prophylactic mastectomy patients shares a similar profile, British journal of cancer 102 (8) (2010) 1284-1293.

[63] W. S. Liang, T. Dunckley, T. G. Beach, A. Grover, D. Mastroeni, D. G. Walker, R. J. Caselli, W. A. Kukull, D. McKeel, J. C. Morris, et al., Gene expression profiles in anatomically and functionally distinct regions of the normal aged human brain, Physiological genomics 28 (3) (2007) 311-322.

[64] W. S. Liang, E. M. Reiman, J. Valla, T. Dunckley, T. G. Beach, A. Grover, T. L. Niedzielko, L. E. Schneider, D. Mastroeni, R. Caselli, et al., Alzheimer's disease is associated with reduced expression of energy metabolism genes in posterior cingulate neurons, Proceedings of the National Academy of Sciences 105 (11) (2008) 4441-4446.

[65] B. S. Paugh, C. Qu, C. Jones, Z. Liu, M. Adamowicz-Brice, J. Zhang, D. A. Bax, B. Coyle, J. Barrow, D. Hargrave, et al., Integrated molecular genetic profiling of pediatric high-grade gliomas reveals key differences with the adult disease, Journal of clinical oncology 28 (18) (2010) 3061-3068.

[66] B. M. Costa, J. S. Smith, Y. Chen, J. Chen, H. S. Phillips, K. D. Aldape, G. Zardo, J. Nigro, C. D. James, J. Fridlyand, et al., Reversing hoxa9 oncogene activation by pi3k inhibition: epigenetic mechanism and prognostic significance in human glioblastoma, Cancer research 70 (2) (2010) 453-462.

[67] H. S. Phillips, S. Kharbanda, R. Chen, W. F. Forrest, R. H. Soriano, T. D. Wu, A. Misra, J. M. Nigro, H. Colman, L. Soroceanu, et al., Molecular subclasses of high-grade glioma predict prognosis, delineate a pattern of disease progression, and resemble stages in neurogenesis, Cancer cell 9 (3) (2006) 157-173.

[68] H. Lockstone, L. Harris, J. Swatton, M. Wayland, A. Holland, S. Bahn, Gene expression profiling in the adult down syndrome brain, Genomics 90 (6) (2007) 647-660.

[69] E. O. Berge, S. Knappskog, S. Geisler, V. Staalesen, M. Pacal, A.-L. Borresen-Dale, P. Puntervoll, J. R. Lillehaug, P. E. Lonning, Identification and characterization of retinoblastoma gene mutations disturbing apoptosis in human breast cancers, Mol Cancer 9 (2010) 173 . 